\title{
Highly Accurate Derivation of the Electron Magnetic Moment Anomaly From Spherical Geometry Using a Single Evaluation
}

\author{
Andrew Worsley ${ }^{1} \&$ James F. Peters ${ }^{2}$ \\ ${ }^{1}$ University College London, UK \\ ${ }^{2}$ University of Manitoba, Canada \\ Correspondence: Andrew Worsley, University College London, UK. E-mail: andrew.worsley@uclmail.net
}

Received: November 4, 2021

Accepted: December 16, 2021

Online Published: January 4, 2022

doi:10.5539/apr.v13n3p30

URL: https://doi.org/10.5539/apr.v13n3p30

\begin{abstract}
The electron magnetic moment anomaly $\left(a_{e}\right)$, is normally derived from the fine structure constant using an intricate method requiring over 13,500 evaluations, which is accurate to $11 \mathrm{dp}$. This paper advances the derivation using the fine structure constant and a spherical geometric model for the charge of the electron to reformulate the equation for $a_{e}$. This highly accurate derivation is also based on the natural $\log \mathrm{e}^{\pi}$, and the zero-order spherical Bessel function. This determines a value for the electron magnetic moment anomaly accurate to 13 decimal places, which gives a result which is 2 orders of magnitude greater in accuracy than the conventional derivation. Thus, this derivation supersedes the accuracy of the conventional derivation using only a single evaluation.
\end{abstract}

Keywords: Bessel function, electron magnetic moment anomaly, electron charge, fine structure constant, geometry, natural $\log$

\section{Introduction}

We have previously published a model for the electron using the conventional equation for the charge of the electron based on spherical geometry (Worsley, 2011). Using this spherical model, we have also previously derived a provisional equation for the electron magnetic moment anomaly (Worsley, \& Peters, 2018). In this paper, further important progress on this work has been made, using only a single evaluation.

In quantum mechanics, the electron magnetic moment anomaly (Kusch, \& Foley, 1947) is related to the electron $g$ factor and the electron magnetic moment to Bohr magneton ratio, by the following expression:

$$
g / 2=-\mu_{e} / \mu_{B}=1+a_{e},
$$

where $g$ is the electron $g$ factor, $\mu_{e}$ is the electron magnetic moment, $\mu_{B}$ is the Bohr magneton and $\mathrm{a}_{\mathrm{e}}$ is the electron magnetic moment anomaly.

The $g$ factor represents a small deviation from the equation for the Bohr magneton. Ordinarily the $g$ factor of the electron (Hanneke, Fogwell, \& Gabrielse, 2008) is derived from $\alpha$, the fine structure constant, using an infinite progression:

$$
g=2\left[1+C_{2}\left(\frac{\alpha}{\pi}\right)+C_{4}\left(\frac{\alpha}{\pi}\right)^{2}+C_{6}\left(\frac{\alpha}{\pi}\right)^{3}+C_{8}\left(\frac{\alpha}{\pi}\right)^{4}+C_{10}\left(\frac{\alpha}{\pi}\right)^{5} \ldots \ldots \ldots\right]_{\mathrm{a} u}+\mathrm{a}_{\text {hadronic }}+\mathrm{a}_{\text {weak }}
$$

The principal term is $\alpha$, and the first variable component $\mathrm{C}_{2}=1 / 2$. The second variable component $C_{4}$ for the electron, is computed empirically using the Riemann zeta function $\zeta_{(x)}$ (Hanneke, Fogwell, \& Gabrielse, 2008), and is given by: 


$$
C_{4}=\frac{197}{144}-\left(\frac{1}{2} \pi \ln 2\right)+\frac{\pi^{2}}{12}+\frac{3}{4} \zeta_{(3)}=-0.328478965579193 \ldots
$$

The third variable component $C_{6}$ is also computed empirically using the Riemann zeta function (Laporta, \& Remmidi, 1996), and is given by:

$$
\begin{aligned}
C_{6}= & \frac{83}{72} \pi^{2} \zeta_{(3)}-\frac{215}{24} \zeta_{(5)}+\frac{100}{3}\left[\left(\sum_{n=1}^{\infty} \frac{1}{2^{n} n^{4}}+\frac{1}{24} \ln ^{4} 2\right)-\frac{1}{24} \pi^{2} \ln ^{2} 2\right] \\
& -\frac{239}{2160} \pi^{4}+\frac{139}{18} \zeta_{(3)}-\frac{298}{9} \pi^{2} \ln 2+\frac{17101}{810} \pi^{2}+\frac{28259}{5184}=1.181241456 \ldots,
\end{aligned}
$$

The fourth term $\mathrm{C}_{8}$ is of enormous complexity, and is derived from a semi analytical evaluation, with 891 evaluations in 4 loop QED, each decomposed into 334 master integrals. This process has taken over 20 years of computation, whose value has recently been revised to -1.912246 (Laporta, 2017). Greater theoretical accuracy is achieved in the standard model with the further addition of $\mathrm{C}_{10}$, using a similar numerical integration technique requiring 12,672 evaluations, which is yet incomplete, and whose approximate value is $9.16(58)$, with a wide error margin (Aoyama, Hayakawa, Kinoshita, \& Nio, 2017).

These evaluations require further adjustment with a number of mass-dependent terms, and the additional coupling factors, $a_{\mu \tau}+a_{\text {hadronic }}+a_{\text {weak }}$. This brings the most recent (2017) theoretical result based to: $1+a_{e}=$ 1.001159652181643 (763), with a wide error margin (Aoyama, Hayakawa, Kinoshita, \& Nio, 2017). As a result, compared to the CODATA (2018) value of 1.00115965218128 (18), this theoretical value is only accurate to $11 \mathrm{dp}$.

Importantly, in this paper the value for $1+a_{e}$ is derived using the conventional equation for the charge of the electron which has been reformulated to give a formula for three spheres based on the speed of light (Worsley, 2011; Worsley, \& Peters, 2018). This model has been improved to produce a more concise formula with superior accuracy by using both the natural $\log e^{\pi}$, and the zero-order spherical Bessel function. Here, this progress has been made which gives a value which is accurate to 13 d.p. using a single evaluation, compared to the conventional derivation of electron magnetic moment anomaly, which requires over 13,500 complex evaluations. This gives a result which is 2 orders of magnitude greater in accuracy than the conventional derivation. Thus this evaluation supersedes the conventional derivation of the electron magnetic moment anomaly.

\section{Methods}

All mathematical calculations follow strict standard algebraic and standard mathematical rules. All derived values are given to 14 decimal places. The principal physics proofs are based upon standard physical and geometrical formulae. The value for the fine structure constant used $\alpha=0.0072973525710$, is taken from the most recent standard experimental method using Caesium-133 atoms in a matter wave interferometer, value $\alpha=$ 0.0072973525713 (14) (Parker, et al., 2018). The value used for Planck's constant $h=6.62607015$ (exact), is taken from the most recent 2018 CODATA evaluation.

\section{Results}

\subsection{Results: Reformulating the Electron Charge}

In standard QED, the charge of the electron is given as:

$$
e^{2}=\varepsilon_{0} .2 h c \alpha
$$

where $e$ is electron charge, $\alpha$ is the fine structure constant, $h$ is Planck's constant, $c$ is the speed of light, and $\varepsilon_{0}$ is the electric constant (permittivity of free space).

The spherical symmetry of the electron charge is well established to a high degree of accuracy (Baldomir, \& Hammond, 2002; Seiden, 2005). Additionally, the fractional quantum Hall effect demonstrated by a magnetic field applied to a 2D electron gas shows that the charge of the electron exists in multiples of 1/3e (Laughlin, 1982; de-Picciotto, Reznikov, Heiblum, et al. 1997). From there we have constructed a model of the electron using three orthogonal spheres rotating in the $\mathrm{x}, \mathrm{y}$ and $\mathrm{z}$ axes. The volume within a sphere is $4 / 3 \pi r^{3}$, and for three spheres the volume would be $3 \times 4 / 3 \pi r^{3}$. 
It has previously been shown that a threefold spherical model of the electron can be derived where the radius is dictated by the speed of light (Worsley 2011). Thus, we can reformulate the standard equation to give the charge of the electron in terms of the volume of three spheres, the electric constant and the speed of light.

$$
e^{2}=\frac{\varepsilon_{0}}{3\left(4 / 3 \pi c^{3}\right)} \cdot 8 \pi h c^{4} \alpha
$$

This equation can be simplified to the term

$$
e^{2}=\frac{\varepsilon_{0}}{3\left(4 / 3 \pi c^{3}\right) \cdot x}
$$

As we have used the conventional equation for the electron charge this agrees with the dimensions of the charge of the electron. So in Eqs. $(6,7)$, the charge of the electron $e$ has the standard dimensions of coulombs $[C]$.

The important aspect of Eq. (7) is that the standard equation for the charge of the electron can be reformulated into two terms. The principal term derives from the volume of three spheres based on the speed of light and the

electric constant, $e^{2}=\frac{\varepsilon_{0}}{3\left(4 / 3 \pi c^{3}\right)}$. This geometric model is corroborated by the experimental localization of single fractional quasi electron charge on the submicron level (Martin, Ilani, Verdene, et al. (2004). Importantly, the second term of this model represents a small spin coefficient, $x=1 / 8 \pi h c^{4} \alpha=1.018722667023144$. This spin coefficient, which is derived from the spherical model of the electron, can then be used to derive an entirely dimensionless term for the electron magnetic moment anomaly (see Section 3.2).

\subsection{Results: Deriving the Electron Magnetic Moment Anomaly}

Here, the derivation of the electron magnetic moment anomaly can be enhanced using a reformulation of the conventional equation for the electron charge [Eqs. (5-7)]. This derivation yields a term from spherical model of the electron, giving the electron spin coefficient $x$, where $x=1 / 8 \pi h c^{4} \alpha$. From here, using a straight forward mathematical cancellation technique, allows the derivation of a completely dimensionless term for the electron magnetic moment anomaly. Since the term $x$ results from derivation of the charge of the electron squared, for the charge of a single electron the spin coefficient $\mathrm{y}$, should be derived from the square root of $x$, hence:

$$
y=x^{1 / 2}-1=\left(1 / 8 \pi h c^{4} \alpha\right)^{1 / 2}-1
$$

This gives the numerical value for $\mathrm{y}=0.009317921679361$ to 14 decimal points. Although the term $x$ and in turn $y$ have dimensions, it is stressed that it is possible to derive an equation which is entirely dimensionless by using the term $y / y$, as in Eq. (12) below, thereby cancelling the dimensions, and producing a dimensionless quantity.

In electromagnetism, an essential dimensionless component is the zero order spherical Bessel function (Bessel 1824), which needs to be incorporated into the equation for greater accuracy. The zero order Bessel function is a universal constant conventionally given as:

$$
J_{0}( \pm i)=I_{0}( \pm 1)=1.266065877752008335598244 \ldots \ldots,
$$

For the plot of $J_{0}$ and $I_{0}$, see Figure 1, computed using the Bessel functions $I_{v}(x)$ and $J_{v}(x)$.

$$
I_{v}(x)=\left(\frac{x}{2}\right)^{v} \sum_{k=0}^{\infty} \frac{\left(\frac{x^{2}}{4}\right)^{k}}{k ! \Gamma(v+k+1)}
$$




$$
J_{v}(x)=\sum_{k=0}^{\infty} \frac{(-1)^{k}\left(\frac{x^{2}}{2}\right)^{y+2 k}}{k !(v+k) !}
$$

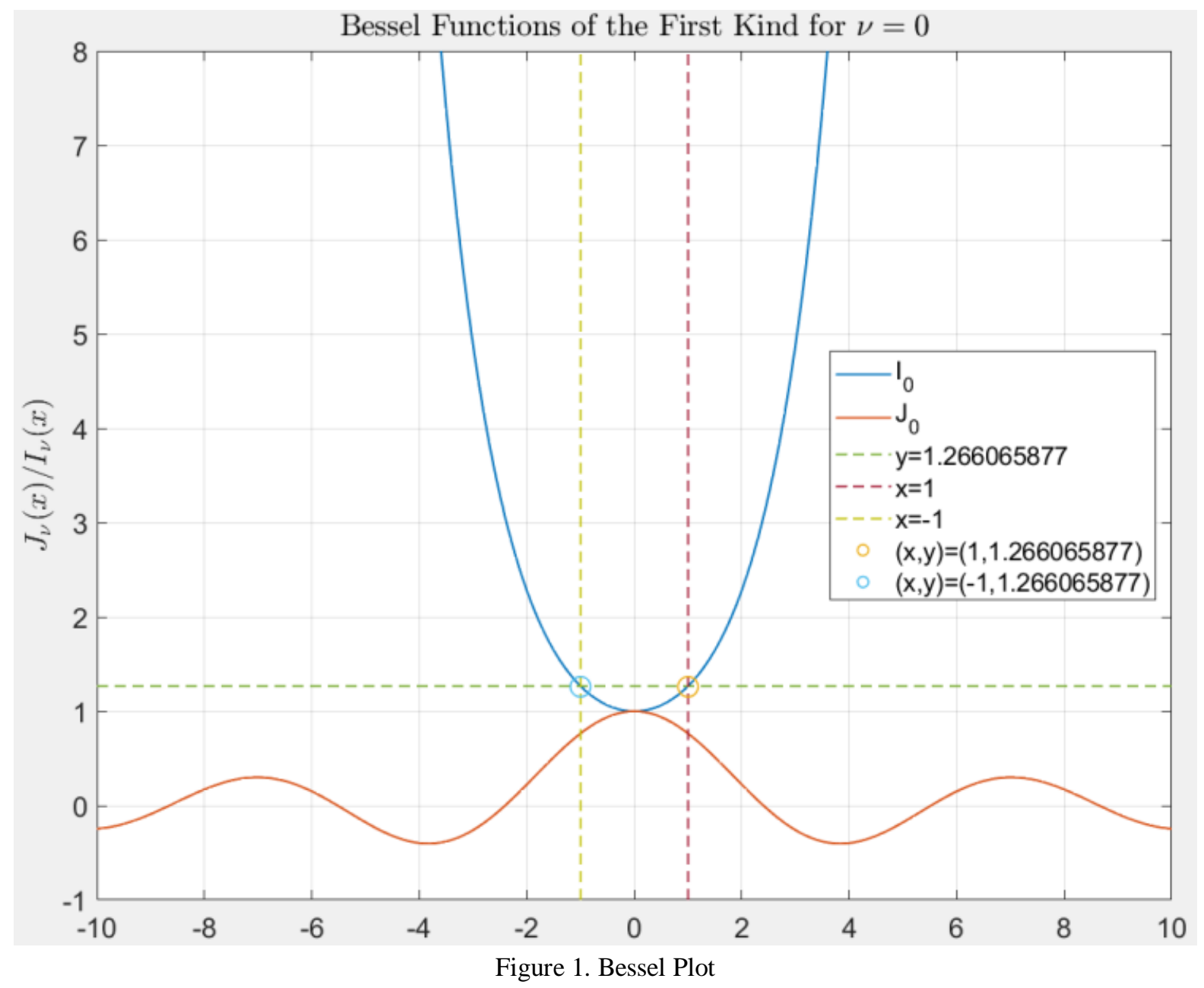

For brevity, this spherical Bessel function value has been designated as:

$$
z=1.266065877752008 \text {. }
$$

Additionally, in accordance with particle gauge coupling (Evans, French., Jensen \& Threlfall, 2010) the ratio in curved space $\left(\mathrm{r}_{\mathrm{w}} / \mathrm{r}\right)^{8}$, gives the power ratio of the electron magnetic moment anomaly $\left(1+a_{e}\right)^{8}$. Thus a far more straight forward dimensionless formula for the anomalous electron magnetic moment, using a single evaluation based on spherical geometry, can be derived.

For the electron magnetic moment anomaly $\left(a_{e}\right)$ :

$$
\frac{y}{\left[\frac{y}{e^{\pi} z}\right]+1}+1=\left(1+a_{e}\right)^{8}
$$

Conventionally this gives a dimensionless parameter, where the electron magnetic moment anomaly principally depends on the term $\mathrm{y}$, derived from conventional formula for the charge of the electron, the natural $\log e^{\pi}$, and the zero order Bessel function $\mathrm{z}$. 
Taking the eight root of Eq. (12). This gives a dimensionless value for the electron magnetic moment anomaly:

$$
1+a_{e} \quad=1.00115965218133(14)
$$

The experimental CODATA (2018) value for the electron magnetic moment anomaly is:

$1+\mathrm{a}_{\mathrm{e}}=1.00115965218128$ (18). This value agrees to 13 d.p. with the single concise term given in Eqs. $(12,13)$, with overlapping error bars. Importantly, this result is 2 orders of magnitude greater in accuracy the conventional derivation. The use of the additional coupling factors, in particular $\mathrm{a}_{\text {weak }}$ (as in the standard model), would readily increase the accuracy further.

\section{Discussion and Conclusion}

With direct concepts of geometry, it possible to advance the derivation of the electron magnetic moment anomaly $\left(\mathrm{a}_{\mathrm{e}}\right)$, from spherical geometry and the term $e^{\pi}$. In this paper, this derivation is considerably advanced by also using the zero order Bessel spherical function commonly used in electromagnetism. This is done using a single evaluation based on the spherical model of the electron charge. The conventional derivation of the electron magnetic moment anomaly (Aoyama, Hayakawa, Kinoshita, \& Nio, 2017) is only accurate to 11 d.p., and requires over 13,500 evaluations. Importantly, in this paper a superior degree of accuracy to 13 d.p. is obtained, with only a single evaluation as in Eq. (12).

Firstly, it is possible to explain the electron's charge in terms of the volume of three spheres and the speed of light. This reformulation of the elementary charge then yields an electron spin coefficient. From here, a concise equation is used to derive the electron magnetic moment anomaly, using Gelfond's constant $e^{\pi}$, where $e^{\pi}$ represents the sum of all unit-ball volumes of even dimensions. As spin depends upon the dimension of time, the electron charge has spin in 4 dimensions, in keeping with previous studies of electron entanglement in 4D hyperspace (Peters, \& Tozzi, 2016). So the derivation is now explained on first geometrical principles.

In addition to its deeper mathematical significance, it appears that Planck's constant $h$ has a profound physical significance, and that Planck's constant and the speed of light $c$ are intimately interconnected, not just with each other, but with the very nature of matter itself. Indeed it is possible to redefine a minimum quantum mass $m_{q}=h / c^{2}$. This minimum quantum mass can also be used to further explain the equations for quantum electrodynamics (Worsley, 2010). This work also brings us closer to the understanding of electrons and their putative formation from photons (Dennis, King, Jack, O'Holleran, et al., 2010; Pike, O. J., et al. 2014; Basdevant \& Dalibard, 2006).

Interestingly, the progression from a three loop evaluation completed in 1996 (Laporta S. \& Remmidi E. 1996), took a further 20 years of computation to progress to the completion of a 4 loop evaluation (Laporta S. (2017). Equally, the 5 loop evaluations are as yet incomplete (Aoyama T., Hayakawa M., Kinoshita T., Nio M. 2017). In the model presented here the computation would be far more rapid as it only requires a single evaluation. Additionally, the increased accuracy of the equation for $\left(\mathrm{a}_{\mathrm{e}}\right)$, is at least in part due to Planck's constant given by CODATA, which has fixed the value of this constant aligned with a fixed value for the electron charge. This means that value derived is not only more accurate but it is also potentially more robust. Greater accuracy will be possible once the fine structure constant is more accurately known. However, this is required to be defined by experimental methods (rather than theoretical methods), with the conventional use of the highly stable Caesium-133 atom (Parker, et al., 2018).

\section{References}

Aoyama, T., Hayakawa, M., Kinoshita, T., \& Nio, M. (2017). Tenth-order electron anomalous magnetic moment: Contribution of diagrams without closed lepton loop. Phys. Rev. D., 96, 019901. https://doi.org/10.1103/PhysRevD.96.019901

Baldomir, D., \& Hammond, P. (2002). Geometry of Electromagnetic Systems. Oxford University Press.

Basdevant, \& Dalibard. (2006). The Quantum Mechanics Solver (2nd ed.). Springer-Verlag Berlin.

Bessel. (1824). Untersuchung des theils der planetarischen störungen, welcher aus der bewegung der sonne entsteht. Berlin: Berlin Math. 
Dennis, M. R., King, R. P., Jack, B., O’Holleran, B., \& Padgett, M. J. (2010). Isolated optical vortex knots. Nature Physics, 6, 118-121. https://doi.org/10.1038/nphys1504

de-Picciotto, R., Reznikov, M., Heiblum, M., Umansky, V., Bunin, G., \& Mahalu, D. (1997). Direct Observation of a Fractional Charge. Nature, 389, 162-164. https://doi.org/10.1038/38241

Evans, N., French, J., Jensen, K., \& Threlfall, E. (2010). Hadronization at the Ads wall. Phys. Rev. D., 81, 066004. https://doi.org/10.1103/PhysRevD.81.066004

Hanneke, D., Fogwell, S., \& Gabrielse, G. (2008). New Measurement or the Electron magnetic Moment and the Fine Structure Constant. Phys. Rev. Lett., 100, 120801. https://doi.org/10.1103/PhysRevLett.100.120801

Kusch, P., \& Foley, H. M. (1947). Precision measurements of the ratio of the atomic 'g Values' in the ${ }^{2} P_{3 / 2}$ and ${ }^{2} P_{1 / 2}$ states of Gallium. Phys. Rev., 72, 1256-1257. https://doi.org/10.1103/PhysRev.72.1256.2

Laporta S. (2017). High Precision Calculation of the 4 Loop Contribution to the Electron g-2in QED. Phys. Lett. B., 772, 232. https://doi.org/10.1016/j.physletb.2017.06.056

Laporta, S., \& Remmidi, E. (1996). The analytical value of the electron (g -2) at order $\alpha^{3}$ in QED. Phys. Lett. B., 379, 283-291. https://doi.org/10.1016/0370-2693(96)00439-X

Laughlin, R. B. (1982). Anomalous Quantum Hall Effect: An Incompressible Quantum Fluid with Fractionally Charged Excitations. Phys. Rev. Lett., 50, 1395-1398. https://doi.org/10.1103/PhysRevLett.50.1395

Martin, J., Ilani, S., Verdene, B., \& et al.. (2004). Localization of Fractionally Charged Quasi Particles. Science, 305(5686), 980-983. https://doi.org/10.1126/science.1099950

Parker R. H., \& et al.. (2018). Measurement of the fine-structure constant as a test of the Standard Model. Science, 360(6385), 191-195. https://doi.org/10.1126/science.aap7706

Peters, J., \& Tozzi, A. (2016). Quantum entanglement on a hypersphere. Int. J. Theo. Phys., 55, 3689-3696. https://doi.org/10.1007/s10773-016-2998-7

Pike, O. J., \& et al.. (2014). A photon -photon collider in a vacuum hohlraum. Nature Photonics, 8, 496. https://doi.org/10.1038/nphoton.2014.95

Seiden, A. (2005). Particle Physics, A Comprehensive Introduction. Addison Wesley, San Francisco.

Worsley, A. (2010). The formulation of harmonic quintessence and a fundamental energy equivalence equation. Phys. Essays, 23, 311-319. https://doi.org/10.4006/1.3392799

Worsley, A. (2011). Harmonic quintessence and the derivation of the charge and mass of the electron and the proton and the quark masses. Phys. Essays, 24, 240-253. https://doi.org/10.4006/1.3567418

Worsley, A., \& Peters, J. (2018). Enhanced Derivation of the Electron Magnetic Moment Anomaly from the Electron Charge using Geometric Principles. Applied Phys. Res., 10(6), 24-28. https://doi.org/10.5539/apr.v10n6p24

\section{Copyrights}

Copyright for this article is retained by the author(s), with first publication rights granted to the journal.

This is an open-access article distributed under the terms and conditions of the Creative Commons Attribution license (http://creativecommons.org/licenses/by/4.0/). 